\title{
LES \\ MICROCENTRALES \\ DE LA MAYENNE
}

\author{
par \\ F. AUROY *
}

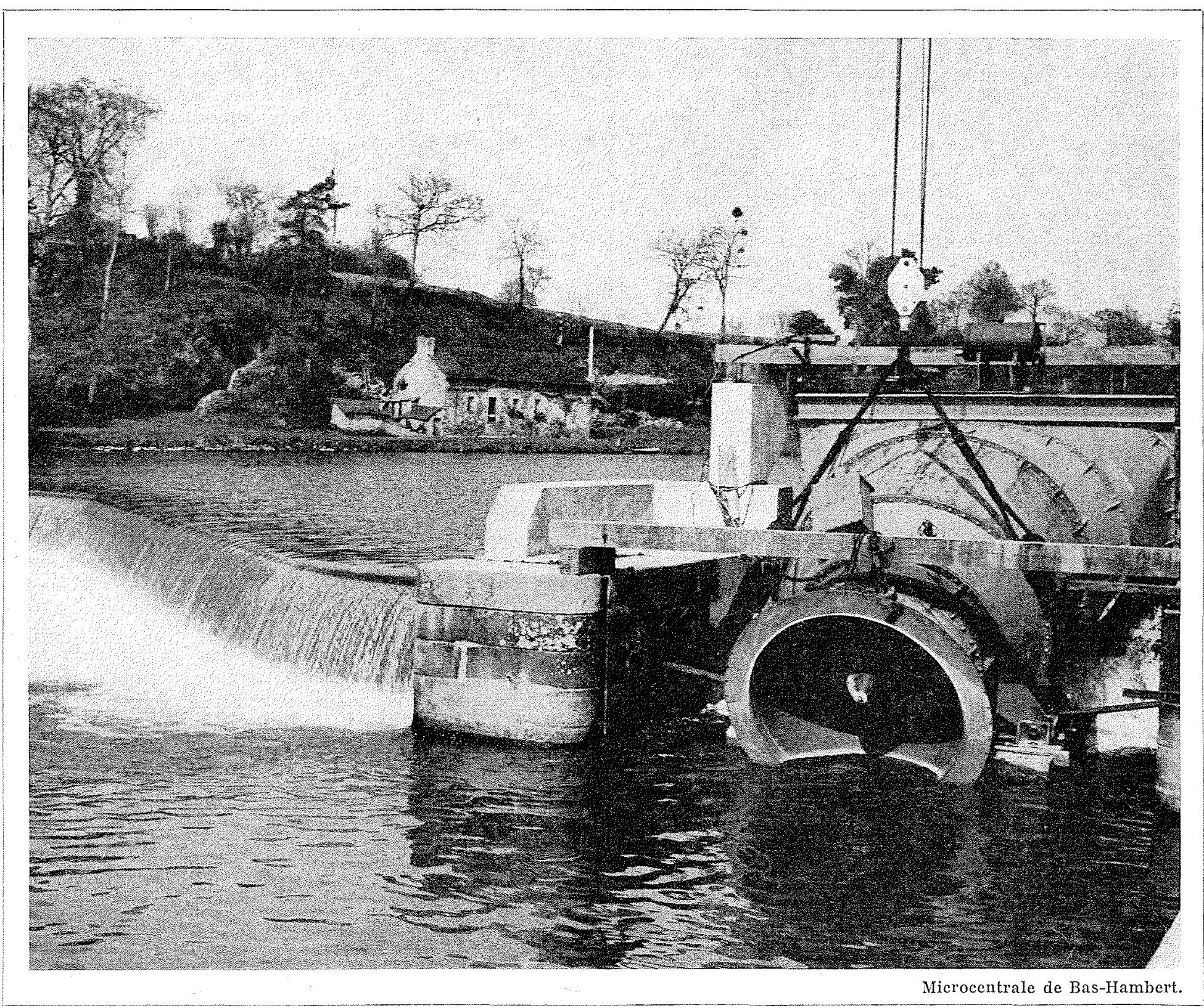

Le terme de « Microcentrales » s'applique à l'équipement de très basses chutes en rivière avec de petits débits; il couvre une gamme de hauteurs de chute de 1 à $5 \mathrm{~m}$ et une gamme de puissances allant de quelques dizaines à un petit nombre de centaines de $\mathrm{kW}$.

Il existe encore, sur les cours d'eau de plaine, de nombreuses petites chutes, dont l'équipement s'était révélé jusqu'ici peu rentable du fait du prix

" Directeur Régional de l'Equipement - Electricité de France. élevé de l'installation vis-à-vis de la puissance disponible. C'est le cas de nombreux moulins et de petites usines dont l'équipement serait à renouveler. C'est le cas aussi de certains biefs de rivières canalisées.

Du point de vue économique, plus la chute est basse, plus le prix de revient au $\mathrm{kW}$ installé est élevé. D’autre part, plus la chute est basse, plus la production est affectée par la diminution de la hauteur de chute due au passage des crues. 
II résulte de ces deux considérations que, pour assurer la rentabilité de tels aménagements, il faut que la durée de fonctionnement soit d'autant plus grande que la chute est plus faible, ce qui implique que plus la chute est basse, plus elle doit être sous-équipée. D'après les calculs de M. de Verdelhan, les optimums seraient les suivants :

\begin{tabular}{|c|c|}
\hline $\begin{array}{c}\text { HaUteur de chute } \\
(\mathrm{en} \mathrm{m})\end{array}$ & $\begin{array}{c}\text { Eovipenewt } \\
\text { (en modules) }\end{array}$ \\
\hline 2 & 0,3 \\
4 & 0,6 \\
6 & 0,9 \\
8 & 1,2 \\
10 & 1,5 \\
\hline
\end{tabular}

Dans la même optique de rentabilité, il y a lieu évidemment de rechercher les meilleurs prix de revient dans tous les domaines:

a) Ouvrages de génie civil:

Pour les réduire au minimum, il faut autant que possible profiter des barrages existants ou n'avoir que peu de travaux de fondation; la salle des machines doit être supprimée.

b) Matériel :

Il doit être de conception simple et son coût pourra être réduit si l'on peut envisager la cons. truction en série d'un minimum de types normalisés.

\section{c) Transport et montage :}

Pour diminuer les prix de transport et de montage, le matériel doit être léger, convenablement fractionné, de façon à faciliter un transport à pied d'œuvre par des chemins parfois difficiles.

d) Exploitation :

Pour supprimer le personnel d'exploitation, l'installation doit être entièrement automatique et non gardiennée.

\section{e) Entretien :}

Pour réduire les charges d'entretien, le matériel doit être simple et robuste, n'exigeant qu'un entretien courant facile, le gros entretien se faisant en atelier après échange standard.

Le groupe bulbe permet de résoudre au mieux ce problème. Rappelons que, dans un groupe bulbe. la roue de turbine et l'alternateur sont calés sur le même arbre, et l'alternateur se trouve à l'inté. rieur d'une carcasse métallique étanche en forme de bulbe, avec laquelle il fait corps. Le bulbe est entièrement immergé dans la veine liquide qui alimente la turbine, et se trouve en général à l'amont de cette dernière; cette disposition ne crée pas de perte de charge dans le diffuseur, ce qui diminuerait les rendements.

La turbine est en général du type hélice, à pales fixes; il est toutefois commode de prévoir la possibilité de modifier à l'arrêt l'inclinaison des pales, de facon à pouvoir modifier le débit turbinable.

La turbine est précédée d'un distributeur fixe, dont les aubes permettent de maintenir le bulbe à l'intérieur de l'enveloppe extérieure.

A l'intérieur du bulbe, la génératrice tourne, soit dans l'air, soit dans l'huile, plus généralement dans une huile diélectrique fluide maintenue en légère surpression par rapport à l'eau de la veine liquide.

L'huile assure une bonne lubrification des paliers, en même temps qu'elle facilite l'évacuation des pertes de l'alternateur, grâce à l'excellent échange thermique entre l'huile et la veine liquide à travers la carcasse du bulbe.

Depuis la première microcentrale à groupe bulbe, réalisée à Röstin en Poméranie (chute moyenne $3,75 \mathrm{~m}$, débit $6,3 \mathrm{~m}^{3} / \mathrm{s}$ ), de nombreuses expérimentations de microcentrales ont été réalisées, principalement en Allemagne, en Autriche et en France.

On peut classer ces expériences en trois types, selon la disposition des groupes bulbe, « en pertuis », «en conduite» ou «en siphon».

Dans la disposition «en pertuis» (fig. 1), le groupe est entièrement immergé dans le pertuis d'alimentation et l'eau attaque directement le distributeur fixe, puis la roue de turbine.

Dans la disposition «en conduite» (fig. 2), le groupe est entièrement immergé dans la veine liquide qui circule dans la conduite d'alimentation venant du réservoir amont.

Dans ces deux solutions, l'axe du groupe est en général horizontal ou peu incliné sur l'horizontale. Elles ont toutes deux un grave inconvénient : pour isoler le groupe, on est obligé de disposer d'un moyen de fermeture à l'amont et d'un autre à l'aval; la vanne d'arrêt pouvant être d'ailleurs à l'amont ou à l'aval, et l'autre moyen de fermeture pouvant n'être qu'un batardeau mis en place à l'arrêt. Ces deux dispositifs de fermeture sont relativement onéreux.

La troisième disposition, dite « en siphon * (fig. 3), évite cette sujétion : le groupe monobloc est placé dans une conduite métallique formant convergent-divergent, dont l'entrée est coudée pour constituer un siphon qui plonge dans le bief amont et dont la sortie plonge dans le bief aval. Le groupe se trouve placé immédiatement à l'aval du coude du siphon, de façon à se trouver toujours hors d'eau. Pour démarrer et arrêter le groupe, il suffit d'amorçer ou de désamorçer le siphon.

Bien que ne constituant pas une installation avec groupe bulbe à écoulement axial, nous citerons une variante de la disposition en siphon (fig. 4) réalisée sur le canal latéral de la Garonne, disposition où malheureusement le groupe est assez difficilement accessible.

Sur la Mayenne canalisée entre les villes de Mayenne et de Laval, l'Electricité de France a réalisé l'équipement de 16 biefs dont les différences de niveau varient de 1,50 à $2,76 \mathrm{~m}$ (fig. 5). Dans chacun de ces biefs, les ouvrages de navigation comportent de la rive gauche à la rive droite:

- une écluse de navigation;

-un pertuis de vannage d'une largeur de $5,20 \mathrm{~m}$ environ, obturé par une vanne à glissière;

- un barrage à seuil déversant.

Les sas d'écluse étant réservés à la navigation, les microcentrales ont été installées dans les pertuis de vidange.

Trois des biefs se prêtaient à l'installation de deux groupes côte à côte, pouvant absorber chacun $4,5 \mathrm{~m}^{3} / \mathrm{s}$, les treize autres biefs ćtant prévus pour. un groupe de $9 \mathrm{~m}^{3} / \mathrm{s}$; au total : 19 groupes. 

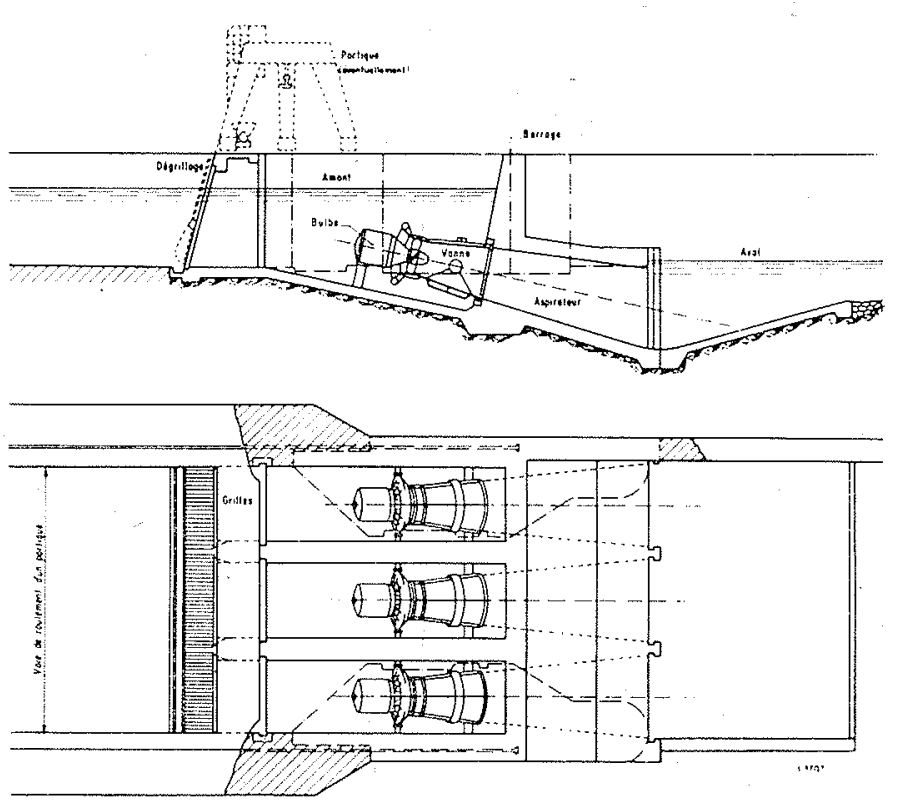

1/ Groupe en pertuis.

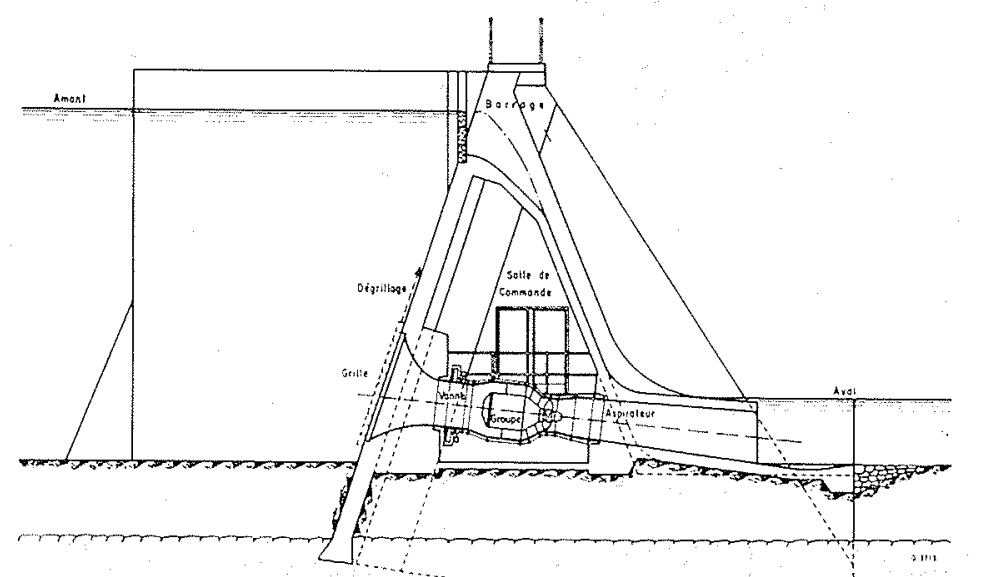

2/ Groupe en conduit.
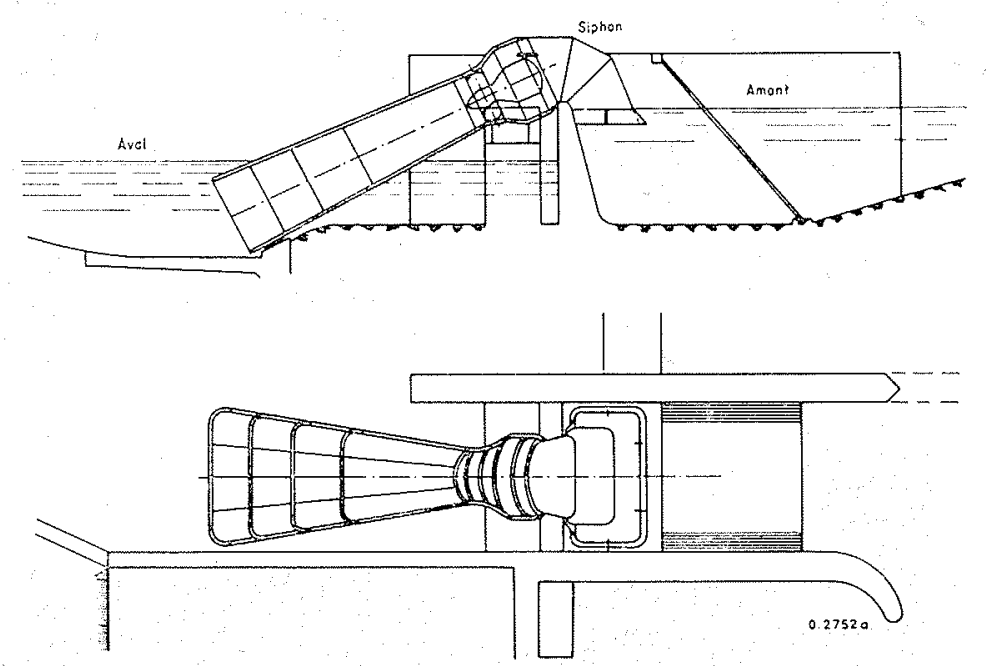

3/ Groupe en siphon.
LA HOUILLE BLANCHE/No $1-1964$

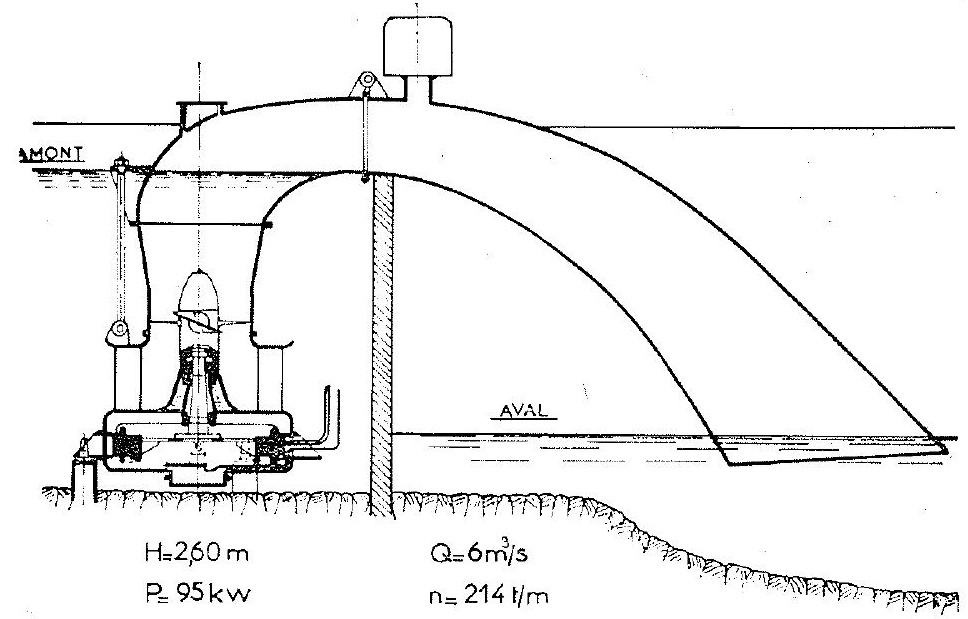

4/ Groupe type Saint-Jorry.

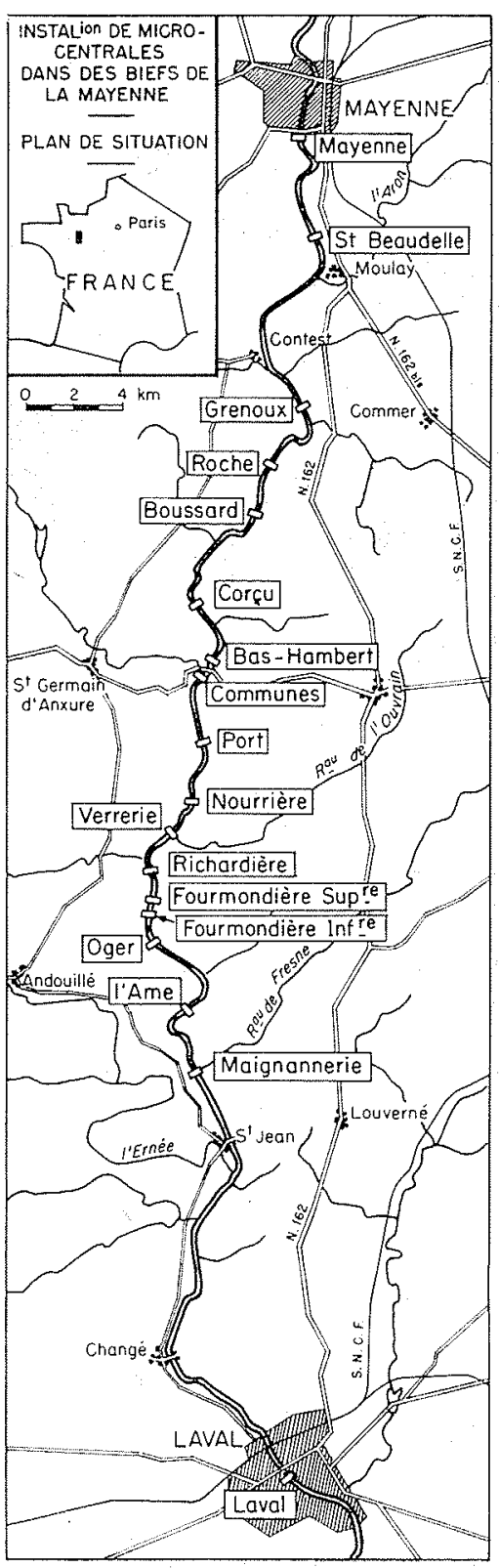

5/ La Mayenne

entre Laval

et Mayenne. 
Notons au passage que le débit moyen annuel de la Mayenne étant d'environ $27 \mathrm{~m}^{3} / \mathrm{s}$, le degré d'équipement de ces microcentrales est bien d'environ 0,3 module.

L'Electricité de France a tout d'abord réalisé el mis en service, en janvier 1954, un premier groupe prototype du type «en siphon» à l'écluse de la Maignannerie (fig. 6), dont les caractéristiques sont :

- débit.

- hauteur de chute.

génératrice asynchrone toumant a

$225 \mathrm{tr} / \mathrm{mn}$, puissance......... $54 \mathrm{~kW}$

L'Electricité de France a, à la même époque, réalisé et mis en service en février 1955, à l'écluse de l'Ame (fig. 7), un groupe prototype du type « en conduite» dont les caractéristiques sont :

- débit . . . . . . . . . . . . $9 \mathrm{~m}^{3} / \mathrm{s}$

-. hauteur de chute.......... $1,90 \mathrm{~m}$;

- génératrice asynchrone tournant à

$225 \mathrm{tr} / \mathrm{mn}$, puissance......... $120 \mathrm{~kW}$.

A la suite de ces deux expériences, l'Electricité de France a décidé d'équiper les autres microcentrales de la Mayenne avec des groupes « en siphon $\gg$. Sur les 17 groupes restant à équiper, dont la puissance va de 50 à $180 \mathrm{~kW}, 12$ sont des groupes asynchrones pouvant absorber chacun $9 \mathrm{~m}^{3} / \mathrm{s}$ et dont l'installation est achevée; les 5 autres sont des groupes synchrones pouvant absorber chacun $4,5 \mathrm{~m}^{3} / \mathrm{s}$ et qui sont en cours d'exécution.

\section{Microcentrales asynchrones.}

Le génie civil de ces microcentrales (fig. 8) est réduit an strict minimum : un simple voile en béton armé au-dessus de la vanne de vidange, qui est une vanne à glissière, manœuvrable à main, de $5 \times 1,17 \mathrm{~m}$. L'ensemble de la microcentrale (siphon, groupe et diffuseur) repose d'une part sur le voile ci-dessus, d'autre part sur deux pilettes en béton armé fondées sur le radier de la passe de vidange; dans certains cas, un petit déroctage dans le lit de la rivière a été nécessaire pour permettre d'asseoir le diffuseur (fig. 9).

Le siphon, qui est calibré pour alimenter le groupe dans les meilleures conditions, constitue en même temps l'organe de coupure de la veine liquide. Il comporte à cet effet, à sa partie supérieure, une ouverture reliée d'une part à une électrovanne et d'autre part à un ventilateur à dépression, par l'intermédiaire d'un clapet de retenue.

Le groupe fonctionne automatiquement par tout ou rien, et le démarrage et l'arrêt sont commandés à l'aide de deux électrodes disposées côté amont, l'une au-dessous de l'autre, à quelques centimètres d'intervalle, à des niveaux réglables.

Quand le plan d'eau atteint le niveau de l'électrode supérieure, un relais provoque la fermeture de l'électrovanne ainsi que la mise en marche du ventilateur. Sous l'effet de la dépression, l'eau monte dans le siphon, puis se déverse vers l'aval, provoquant le démarrage de la turbine. La vitesse du groupe augmente à mesure que le débit augmente, et lorsqu'elle approche de la vitesse de synchronisme une électrode spéciale, détectant le nivean correspondant au débit de marche à vide, provoque le couplage sur le réseau. A ce moment, un relais provoque l'arrêt du ventilateur.
Tant que le débit du groupe est inférieur à celui de la rivière, le groupe tourne et débite sur le réseau. Si le débit de la rivière est inférieur à celui du groupe, le plan d'eau amont baisse, et lorsqu'il atteint l'électrode inférieure, un relais provoque l'ouverture de l'électrovanne ainsi que celle du contacteur principal. Le groupe est découplé et, en même temps, le siphon se désamorce et le groupe s'arrête. Le cycle recommence dès que le plan d'eau amont atteint à nouveau l'électrode supérieure.

Le plan d'eau amont devant être maintenu à une cote au moins égale à celle du barrage, l'électrode inférieure est calće à la cote d'arasement du barrage el l'électrode supérieure est placée immédiatement au-dessus du niveau bas de l'intumescence produite par l'arrêt du groupe.

Les turbines sont des turbines hélices capables d'absorber un débit de $9 \mathrm{~m}^{3} / \mathrm{s}$; elles tournent à $143 \mathrm{tr} / \mathrm{mn}$. Les roues, "de $1,12 \mathrm{~m}$ de diamètre, sont à quatre pales et l'orientation des pales peut être modifiée à l'arrêt.

Les génératrices sont du type asynchrone fonctionnant à la tension de $500 \mathrm{~V}$ et tournent dans une huile en légère surpression grâce à un réservoir spécial placé au-dessus du siphon. Elles présentent une forte réactance, une faible inertie, et peuvent tourner en permanence à la vitesse d'emballement de $435 \mathrm{tr} / \mathrm{mn}$.

Les groupes sont interchangeables, bien que les génératrices soient de deux types différents, selon que la hautenr de chute est inférieure ou supérieure à 2,05 $\mathrm{m}$. Le groupe avec génératrice type EU1250 fournit de 70 à $135 \mathrm{~kW}$ quand la hauteur de chute varie de 1,35 à $2,05 \mathrm{~m}$. Le groupe avec génératrice type EU 1250 bis fournit de 135 à $190 \mathrm{~kW}$ quand la hauteur de chute varie de 2,05 à $2,75 \mathrm{~m}$.

Une grille est disposée à l'entrée du siphon; elle est en forme de secteur mobile autour d'un axe horizontal. Cette disposition est intéressante, car les feuilles et branches qui sont arrêtées par cette grille ont naturellement tendance à retomber à chaque arrêt du groupe, le point de chute se situant exactement devant la vanne de vidange.

L'amenée à pied d'œuvre des éléments de groupes a été faite par chaland à partir du port de Mayenne. Les montages sur place ont été réalisés grâce à un derrick en duralumin facile à démonter et à transporter (fig. 10).

L'énergie produite par chaque microcentrale est évacuée par l'intermédiaire d'un transformateur $500 \mathrm{~V} / 15 \mathrm{kV}$ sur une ligne $15 \mathrm{kV}$ construite à cet effet entre Laval et Mayenne, et raccordée aux barres $15 \mathrm{kV}$ du poste de Mayenne.

Afin de diminuer les pertes en ligne, la puissance réellement absorbée par les génératrices est partiellement compensée sur place par des batteries de condensateurs statiques $500 \mathrm{~V}$, de $90 \mathrm{KVAR}$ ou de $120 \mathrm{KVAR}$, selon la puissance de la microcentrale. 
Un petit bâtiment en béton armé (fig. 11) de $4 \times 2 \mathrm{~m}$ de dimension extérieure, et de $2,50 \mathrm{~m}$ de hauteur, situé sur la rive à proximité immédiate de chaque microcentrale, abrite :

- le transformateur;

- la batterie de condensateurs;

- un jeu de trois fusibles à haut pouvoir de coupure;

- un jeu de trois transformateurs d'intensité;

- un châssis équipé des différents contacteurs (génératrice, batterie de condensateurs, moteur du- ventilateur, électrovanne);

- un châssis de commande et de protection comportant les différents relais de protection suivants : coupure, inversion de phases, minimum et maximum de tension, maximum de fréquence, masse basse tension, temps de démarrage trop long, fatigue.

Cette dernière protection mérite un petit commentaire. On désigne sous le terme de «fatigue» un phénomène qui se traduit par une baisse plus ou moins rapide de la puissance fournie, et qui est $d \hat{u}$ à l'accumulation des feuilles contre la grille ou contre la paroi des pales qui se trouve en dépression. En général, lorsque le groupe s'arrête, les feuilles se détachent d'elles-mêmes et le groupe retrouve sa puissance. Le relais provoque donc la mise à l'arrêt et le redémarrage du groupe.

Le fonctionnement par tout ou rien de ces microcentrales fait qu'elles n'apportent au réseau qu'une énergie d'appoint, intermittente. Le réseau doit donc être en mesure de pallier les périodes de défaillance, ainsi que d'assurer le réglage de la fréquence et de la tension.

\section{Microcentrales synchrones.}

Dans le cadre de la recherche d'un type d'installation pour réseau autonome de faible puissance, utilisable notamment dans les pays en voie de développement, Electricité de France a réalisé et mis en service en 1958 à Echarcon, sur la rivière l'Essonne, une installation expérimentale, puis a décidé en 1961 de réaliser sur la Mayenne cinq installations synchrones, semblables à celle d'Echarcon, qui sont en cours de fabrication.

L'installation d'Echarcon (fig. 12) est une installation en siphon, fonctionnant par tout ou rien en fonction du niveau amont grâce à une électrovanne et à un ventilateur, comme décrit plus hau à propos des microcentrales asynchrones.

A sa partie supérieure, le siphon comporte une trémie de $1 \mathrm{~m}$ de diamètre fermée par un couvercle bombé en stratifié à fermeture autoclave; ce couvercle très léger s'ouvre et se ferme très facilement et permet en conséquence l'accès rapide et facile au bulbe; c'est par cet orifice qu'on sort l'alternateur en cas de démontage (fig. 13).

La turbine est une roue hélice de $1,12 \mathrm{~m}$ de diamètre tournant à $214 \mathrm{tr} / \mathrm{mm}$, à quatre pales fixes, en alliage d'aluminium, dont le moyeu s'encastre dans la partie aval du distributeur fixe.

Le bulbe contenant la génératrice également en alliage d'aluminium vient s'appuyer à l'amont sur le distributeur (fig. 14).
La génératrice est du type synchrone à excitation par aimants permanents.

Le rotor de la génératrice est constitué par une jante sur laquelle sont fixés les blocs d'aimants permanents, chaque pòle étant entouré par un bobinage d'aimantation.

Le stator est constitué par un paquet de tôles empilées dans un circuit en alliage léger coulé.

La génératrice peut fonctionner indifféremment dans l'huile ou dans l'air. La pièce porte-paliers (en alliage léger comme le cuvelage) forme réserve d'huile de graissage des paliers, en légère surpression par rapport à la pression d'eau au droit des joints, de façon à protéger l'alternateur contre les rentrées d'eau éventuelles.

Dans le cas particulier d'Echarcon, le barrage a été réalisé par un voile constitué de tóles métalliques juxtaposées mais non solidaires, fixé d'une part dans leur radier, d'autre part à une poutre horizontale scellée dans les bajoyers sous le col du siphon. L'étanchéité est assurée par des bandes de caoutchouc formant joint entre les toles et entre le voile et les bajoyers.

Le siphon et le diffuseur sont en tôle d'acier de $4 \mathrm{~mm}$ d'épaisseur.

La tension aux bornes de la génératrice est de $220 \mathrm{~V}$ entre phases. Pour une chute de $1,60 \mathrm{~m}$, et un débit de $4,5 \mathrm{~m}^{3} / \mathrm{s}$, la puissance débitée est de $52 \mathrm{~kW}$.

Le poids total de la microcentrale est de $6000 \mathrm{~kg}$.

Dans le cas du fonctionnement dans un réseau interconnecté, la marche d'une telle microcentrale synchrone peut être entièrement automatique comme vu plus haut pour les microcentrales asynchrones.

Dans le cas du fonctionnement en réseau séparé, il faut disposer d'une petite source d'énergie auxiliaire pour la mise en route du ventilateur à dépression.

Les interventions sur place se limitent d'ailleurs aux opérations suivantes :

- dégagement éventuel de corps étrangers ayant échappé à la grille et gênant le fonctionnement de la turbine;

- remplacement de l'huile des paliers;

- graissage du ventilateur;

-- peinture de renouvellement.

En cas d'avarie sur des organes plus importants, le démontage s'effectue en quelques heures à l'aide d'un palan, la pièce la plus lourde, qui est l'alternateur, n'excédant pas $1100 \mathrm{~kg}$.

La réaimantation des aimants permanents se fait aisément en déchargeant une batterie de condensateurs portative dans chaque paire de pôles de la génératrice, les prises terminales des bobines d'aimantation étant facilement accessibles lorsqu'on a enlevé le couvercle supérieur de l'alternateur.

L'appareillage automatique est constitué par des circuits statiques semi-conducteurs), Cet appareillage assume les tâches suivantes:

- amorçage et désamorçage du siphon en fonc- 


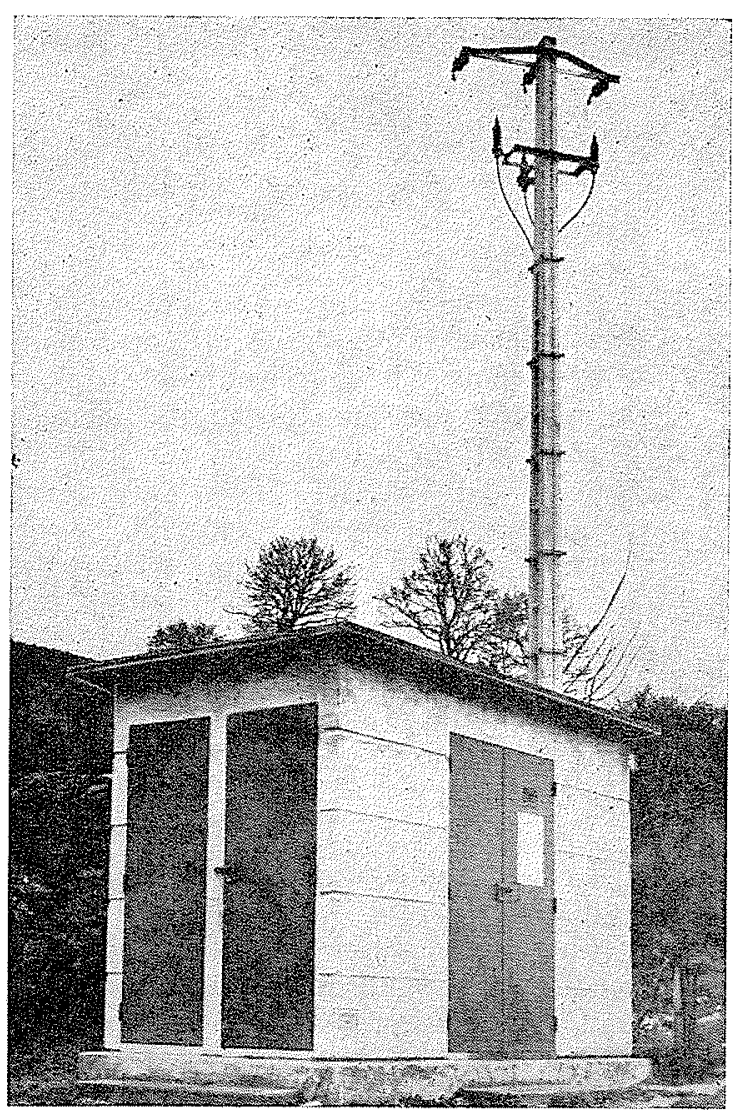

11/
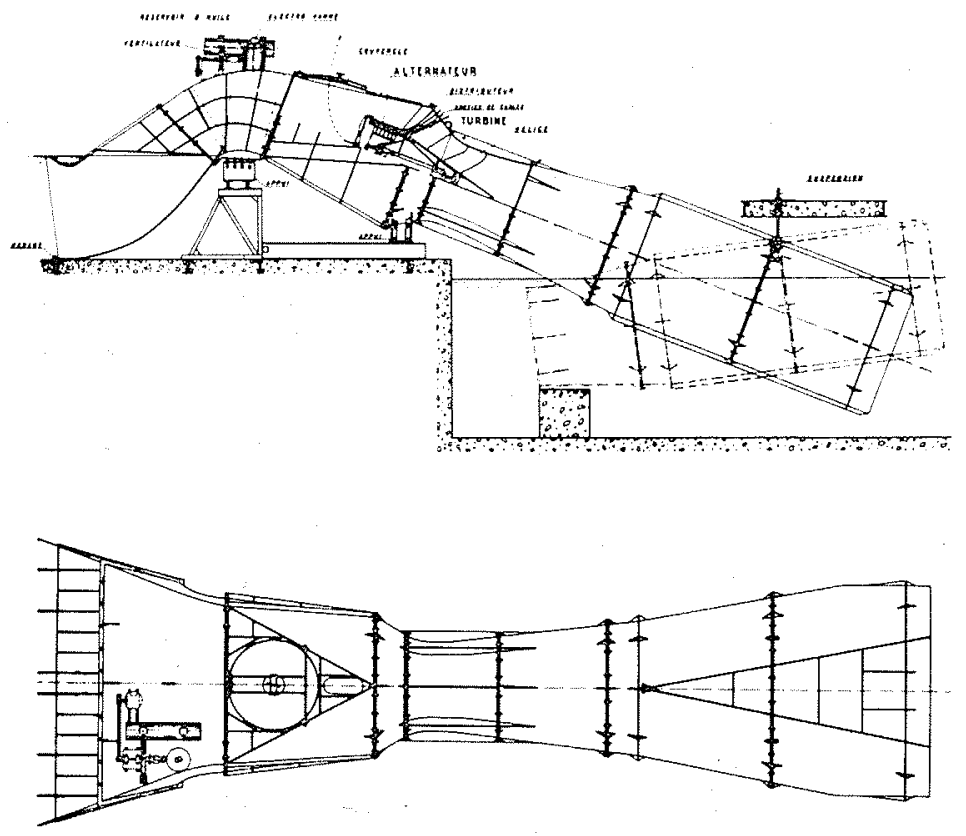

12/ Microcentrale d'Echarcon.

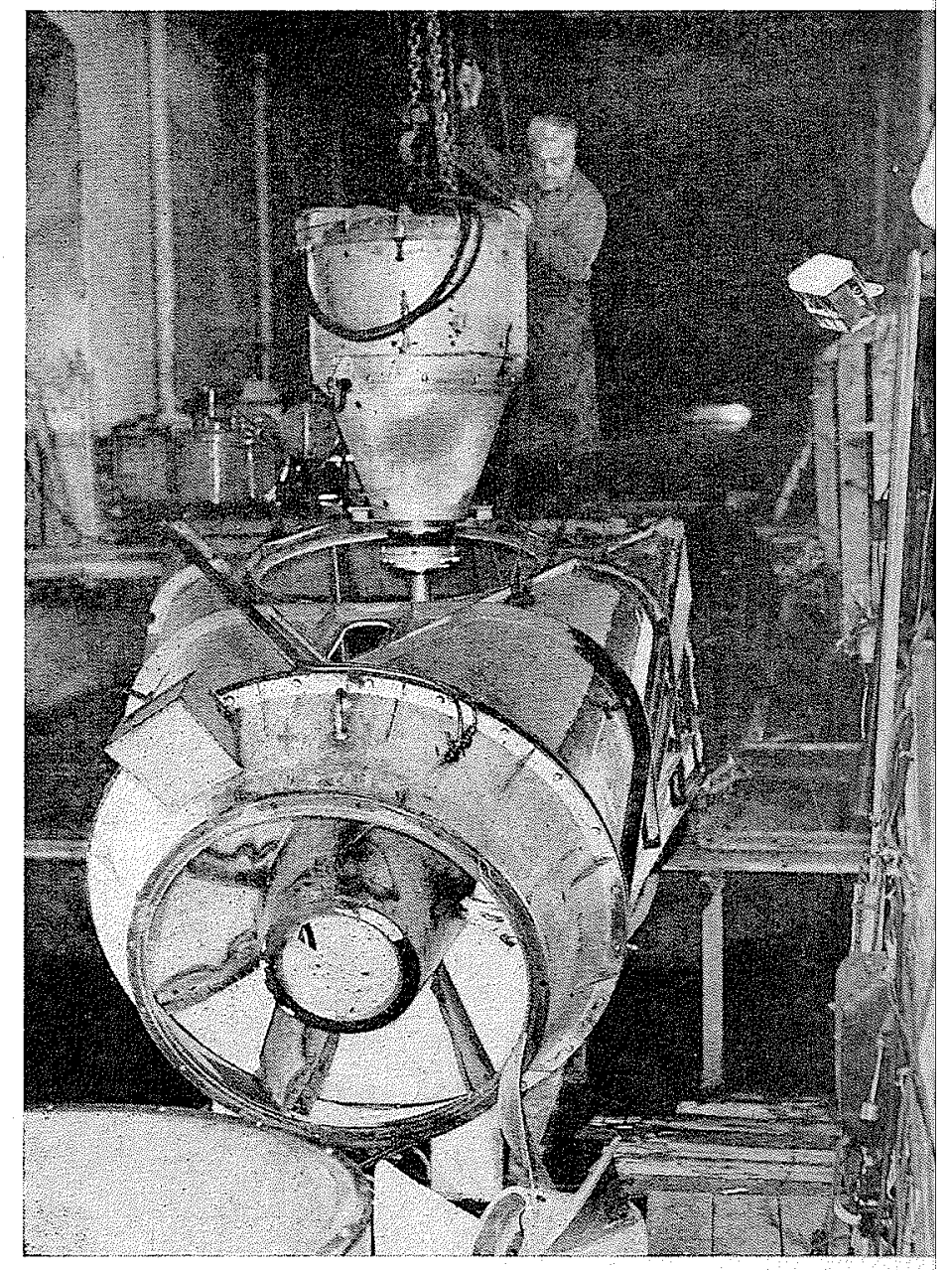

13/ Echarcon. Sortie du moulinet.

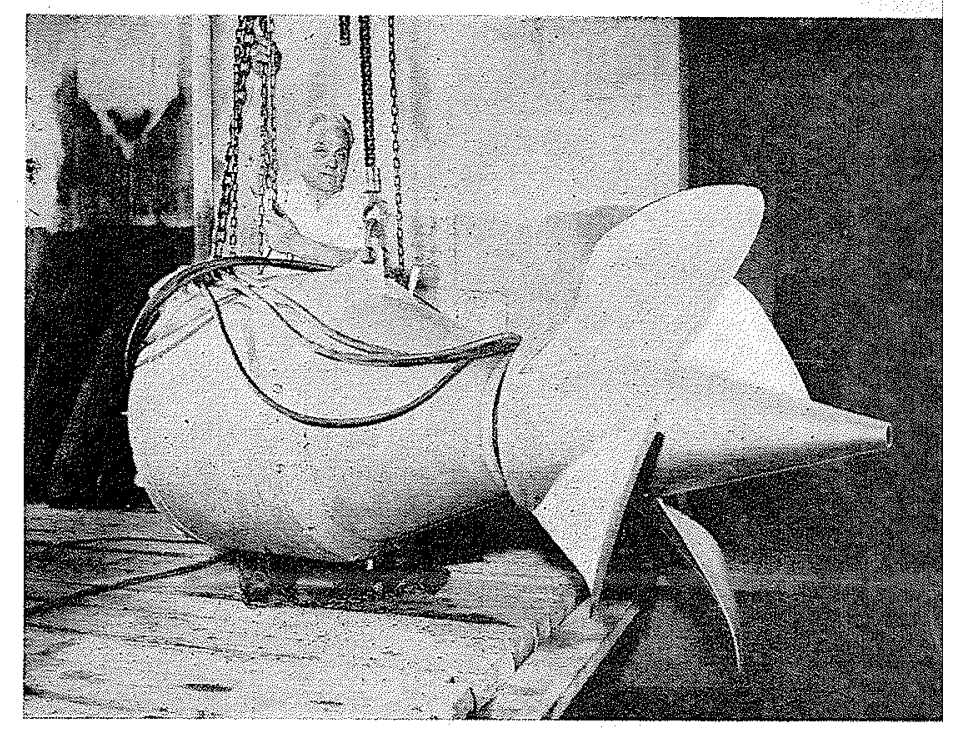

14/ Echarcon. Le moulinet. 
tion du niveau amont;

- couplage de l'alternateur au réseau;

- contrôle du démarrage et réitération du démarrage, limitée à trois tentatives si la puissance fournie est insuffisante;

- protections (protection à maximum et minimum de tension, protection à maximum et minimum de fréquence, protections contre les courts-circuits en ligne, contre les surintensités, contre les défauts de masse, contre les échauffements).

Dans le but de permettre un fonctionnement en réseau autonome, un dispositif de régulation par absorption a été construit et expérimenté à Echarcon, grâce auquel le groupe fonctionne à puissance constante. La puissance qui n'est pas consommée par le réseau est absorbée par des résistances immergées dans le bief aval. Un amplificateur. magnétique contrôle le courant qui traverse les résistances. La puissance absorbée est asservie à la fréquence.

Malgré le manque d'inertie du groupe bulbe, caractérisé par son temps de lancer de $0,5 \mathrm{~s}$, la coupure ou la fermeture instantanées de $90 \%$ de la puissance du groupe ne provoquent qu'une variation transitoire de $\pm 10 \%$ de la fréquence.

La totalité de l'appareillage de la microcentrale est contenue dans une armoire étanche, d'environ
$1 \times 1 \times 0,50 \mathrm{~m}$, placée immédiatement à côté du groupe.

Les cinq machines en cours de fabrication et destinées à la Mayenne diffèrent peu du prototype d'Echarcon. La roue comporte quatre pales fixées au milieu par des tourillons permettant d'ajuster leur position à l'angle voulu pour fixer à la turbine le débit désiré. La vitesse de rotation est de $250 \mathrm{tr} / \mathrm{mn}$; la tension est $220 / 380 \mathrm{~V}$ triphasé.

Dans chaque installation, le barrage est constitué par un simple voile en béton armé entre les bajoyers de la passe de vidange au-dessus de la vanne de vidange, disposition semblable à celle des microcentrales asynchrones.

$$
*
$$

Les microcentrales synchrones ou asynchrones constituent une formule qui peut présenter en France un certain intérêt dans les cas particuliers, où moyennant de faibles dépenses de génie civil on pourra réaliser des installations rentables.

Par contre, les microcentrales synchrones constituent certainement une solution valable dans les pays en voie de développement, employées seules ou en liaison avec de petites centrales thermiques. 